Voix et Images

voixetimages

\title{
Le syndrome de la fin
}

\section{Martine-Emmanuelle Lapointe}

Volume 33, numéro 2 (98), hiver 2008

Michel Beaulieu

URI : https://id.erudit.org/iderudit/018277ar

DOI : https://doi.org/10.7202/018277ar

Aller au sommaire du numéro

Éditeur(s)

Université du Québec à Montréal

ISSN

0318-9201 (imprimé)

1705-933X (numérique)

Découvrir la revue

Citer ce compte rendu

Lapointe, M.-E. (2008). Compte rendu de [Le syndrome de la fin]. Voix et Images, 33(2), 144-149. https://doi.org/10.7202/018277ar

Ce document est protégé par la loi sur le droit d'auteur. L'utilisation des services d'Érudit (y compris la reproduction) est assujettie à sa politique d'utilisation que vous pouvez consulter en ligne.

https://apropos.erudit.org/fr/usagers/politique-dutilisation/
Cet article est diffusé et préservé par Érudit.

Érudit est un consortium interuniversitaire sans but lucratif composé de l’Université de Montréal, l'Université Laval et l'Université du Québec à Montréal. Il a pour mission la promotion et la valorisation de la recherche. https://www.erudit.org/fr/ 


\author{
R O M A N \\ Le syndrome de la fin \\ $+++$ \\ MARTINE-EMMANUELLE LAPOINTE \\ Université de Montréal
}

On ne cesse de l'évoquer. La fin serait là, pas très loin, présence vague et précise à la fois dont l'horizon serait pleinement investi. Inutile de dire que le roman contemporain en porte les traces, qu'il s'inspire des statistiques crépusculaires sur l'état de notre planète, de la vacuité des relations sociales ou de la disparition des grandes idéologies. Le syndrome de la fin, ou du post - si abondamment invoqué dans les médias - travaille, souterrainement ou non, les derniers ouvrages d'Élise Turcotte, de Nelly Arcan et de Stéfani Meunier, et témoigne assez éloquemment d'une sorte d'angoisse diffuse de la disparition.

Élise Turcotte a souvent fait du lieu - mais plus encore de la maison - l'un des personnages privilégiés de ses fictions. Dans Le bruit des choses vivantes ${ }^{1}$, la maison d'Albanie et de Maria constitue un refuge, une cellule à la fois close et ouverte, qui protège des assauts de la vie extérieure et des images apocalyptiques diffusées par les médias. Le lieu et les objets quotidiens de La maison étrangère deviennent littéralement le prolongement du corps et de la conscience d'Élisabeth. Forteresses et havres, les maisons d'Élise Turcotte sont cependant investies par les signes de la perte et contraignent fort souvent le sujet qui les habite à s'y réfugier pour mieux $s^{\prime} y$ égarer. Jamais Élise Turcotte n'aura-t-elle insisté autant sur la négativité du lieu habité que dans son dernier ouvrage, Pourquoi faire une maison avec ses morts ${ }^{2}$, paru tout récemment chez Leméac. Comme son titre l'indique, le récit porte les traces d'une réflexion sur la cohabitation des morts et des vivants, sur cette mince frontière qui les sépare et qui menace à tout moment de s'effacer.

La première phrase de l'ouvrage nous plonge d'emblée dans un univers étrange peuplé de fantômes, d'animaux ou d'humains, de présences évanescentes aussi nombreuses que changeantes: «J'ai toujours trop pensé à la mort. Du plus loin que je

$$
++
$$

1 Élise Turcotte, Le bruit des choses vivantes, Montréal/Arles, Leméac/Actes sud, coll. «Babel», 1998 [1991].

2 Id., Pourquoi faire une maison avec ses morts, Montréal, Leméac, 2007, 124 p. 
me souvienne, elle s'est manifestée à moi sous des formes diverses. » (9) Au fil des sept histoires qui le composent, chapitres d'un roman ou courtes nouvelles interreliées, le récit explore les thématiques du suicide, de la divination, de la lente décomposition des cadavres, de la maladie, des rites funéraires, mais surtout de la fin du monde ou d'un monde qui, aux yeux de la narratrice, s'avère imminente. Pourquoi faire une maison avec ses morts? Cette interrogation, centrale s'il en est, renvoie d'abord à l'absence d'espace réservé aux défunts dans le monde contemporain, voire à l'omniprésence d'une version propre et aseptisée de la mort. La narratrice affirme: «aujourd'hui, plus personne ne sait comment faire une maison avec ses morts » (48), ajoutant un peu plus loin qu' « [o]n se filme pour dire adieu, ainsi quand le dernier jour arrive, le deuil est déjà accompli. C'est une nouvelle loi: nous devons laisser les vivants tranquilles et organiser notre propre disparition» (49). Paradoxalement, c'est en refoulant la mort que l'humanité s'approche le plus dangereusement de sa propre perte, incapable d'interpréter les signes, pourtant manifestes, de sa disparition. Pourquoi faire une maison avec ses morts? La question pourrait également être formulée ainsi : pourquoi s'aveugler devant cette mort que l'on accueille tout de même chez soi, pourquoi la préméditer aveuglément, pourquoi faire en sorte qu'elle advienne sans qu'on y soit préparé? Les signes de la catastrophe ne cessent de se multiplier. La narratrice «sai[t] que la fin de ce monde est proche parce que le récit de sa mort a été coupé» (49). Tant la disparition des espèces animales que les fusillades meurtrières dans les écoles nord-américaines annoncent le grand effondrement, dont l'ultime chapitre, intitulé « Journal d'une mortelle», livre une version librement inspirée du déluge biblique.

Au-delà de cette représentation de la fin du monde, qui ramène aux problématiques de l'écologie et de la responsabilité de l'individu face à sa propre disparition, Pourquoi faire une maison avec ses morts propose également une réflexion sur la transmission des héritages. Comment transmettre lorsque l'avenir même est en voie d'oblitération, lorsque le seul horizon offert aux héritiers ne se présente que sous la forme d'une vague promesse? "Avant, l'enfance ne se tenait pas devant moi comme un arbre brûlé» (95), écrit Élise Turcotte. Aussi éloigné du Bruit des choses vivantes soit-il, le dernier ouvrage de l'auteure présente un dialogue entre les générations et cherche à faire émerger ce qui survit encore, et mérite de s'épanouir, malgré le catastrophisme ambiant. Ce dernier livre exigeant, presque abstrait, ne connaîtra peut-être pas le même succès que les romans précédents d'Élise Turcotte. Néanmoins, il saura sans doute s'imposer au fil du temps, aussi doucement qu'il s'impose à la conscience du lecteur qui, sans savoir exactement où on le mène, accepte sciemment de se laisser travailler par la mort, en autant qu'elle lui permette aussi d'«assiste[r] au maintien de la vie» (115).

La mort habite également $\bar{A}$ ciel ouvert ${ }^{3}$, le dernier roman de Nelly Arcan. Elle emprunte cependant une forme différente, presque opposée à vrai dire aux multiples

$+++$

3 Nelly Arcan, À ciel ouvert, Paris, Seuil, 2007, 271 p. 
mises en scène de la fin du monde de Pourquoi faire une maison avec ses morts. Chez Arcan, la monstration, le désir de paraître, l'obsession de la beauté artificielle, la «burqa de chair», pour reprendre la métaphore de l'auteure, côtoient la folie, la destruction et la violence. Autre climat de fin du monde, autre réflexion sur le malaise contemporain, mais présentés d'une manière ostentatoire, parfois volontairement grossière, qui ramène inévitablement à la superficialité d'un monde où les rapports sociaux s'apparentent à de mauvaises publicités.

L'intrigue repose sur les tribulations d'un triangle amoureux. Julie, scénariste de documentaires, s'est reconstruite après avoir vécu une rupture amoureuse terrible. Sa renaissance s'avère liée à une véritable purgation physique:

la métamorphose [...] qu'elle avait subie; un amaigrissement causé par la drogue, une manière de fuir les regards, de boire trop et seule dans un coin, une réputation de charrue, une impression de saleté mentale, une aura de crasse, de déroute que reflétaient ses yeux, sa peau, puis une lente résurrection du corps, une blondeur encore plus blonde, une tête relevée montrant la santé, une stature plus solide, mieux ancrée, une retenue dans les lieux publics, la dignité retrouvée d'une femme qui avait mal tourné. Une femme tuée par l'amour [...] qui avait réappris à marcher, à respirer (41).

Forte de cette dignité retrouvée, Julie affiche une froideur redoutable qu'elle expose fièrement dans les bars, les clubs de gym et les cafés du Plateau Mont-Royal. Une rencontre sur le toit de son immeuble - d'où le titre $\bar{A}$ ciel ouvert - menace cependant d'ébranler son nouvel équilibre. Elle y croise sa voisine Rose, une styliste de mode, qui arbore comme elle une beauté fabriquée; toutes deux, précise la narration omnisciente, "étaient belles de cette beauté construite dans les privations, elles s'en étaient arrogé les traits par la torsion du corps soumis à la musculation, à la sudation, à la violence de la chirurgie » (17). Avant même de connaître celle qui deviendra sa rivale, Rose la jalouse comme elle jalouse les autres femmes, surtout les belles, qu'elle appelle secrètement «chiennes» (46). Elle soupçonne Julie d'intéresser son conjoint Charles, le beau et doux photographe de mode. C'est la suspicion même de Rose qui fera naître en Julie le désir de séduire Charles. Cette dernière se prêtera d'ailleurs au jeu, comme pour triompher d'un ennui la privant de ses émotions propres. Julie réussit certes à conquérir Charles, mais au prix de sa santé physique et de son équilibre mental car l'amour intense, du moins chez Nelly Arcan, conduit à la folie, à l'abêtissement, à la mort, cela même si l'autre devient «le Regard qui englob[e] tous les autres» (142). Charles, le personnage le plus effacé du roman, sorte de pantin manipulé par ses deux femmes, sombre littéralement dans la folie lorsqu'il découvre que sa sexualité fétichiste s'inspire des traumatismes de son enfance passée dans la boucherie de son père. Dès lors, la beauté sera pour lui «une feuille dorée sur fond sanglant, linceul fleuri qui cach[e] la crudité de la chair et son fonctionnement organique» (232), dévoilant «le visage brandi de la mort» (233).

Comme dans ses deux premiers ouvrages, Nelly Arcan se livre à un examen de la psyché féminine contemporaine et soutient qu'elle est dévorée par le désir de plaire et l'ambition de ressembler aux modèles de "perfection" physique imposés 
par les magazines de mode et les médias. L'homme, ici encore, n'existe que par défaut, presque abstraitement, et se présente tel le représentant d'un regard plus global, commercial pourrait-on dire, qui afflige et stimule à la fois les héroïnes du roman. Ce sont plutôt les femmes qui se regardent vraiment, qui se jaugent et se déchirent afin de satisfaire l'EFil masculin, ou du moins ce qu'elles supposent être l'Eil masculin. Malgré ses défauts apparents, sur lesquels je reviendrai, le roman réussit parfois à exhumer avec finesse, et sans donner dans le moralisme ou dans la dissertation académique, le nauséeux marasme du temps présent. Il met au jour la laideur, la violence d'un monde dominé par la compétition sous toutes ses formes, la dictature du bon goût et la soumission aux canons aveugles de l'industrie. On se dit même à quelques reprises: non, il n'y a pas d'exagération, ce vide, cette béance existent et renvoient à leur manière à un sentiment de fin du monde. Sans être incarné en un phénomène naturel comme chez Élise Turcotte, le sentiment de la fin émerge de situations connues, plane comme une menace au-dessus des conversations oiseuses tenues à la terrasse du Plan B ou au-dessus de n'importe quel toit du Plateau Mont-Royal.

En abandonnant le genre de l'autofiction, l'auteure a sans doute voulu replacer les thématiques qui lui étaient chères dans un cadre romanesque. Malheureusement, ses obsessions deviennent de plus en plus caricaturales au fil du roman. À force de dénoncer le vide ambiant et de vouloir le représenter fidèlement, l'auteure y succombe. Le passage de l'idée - la marchandisation, voire la sexualisation, outrancière du corps - au roman ne s'accomplit pas sans heurts. Les personnages demeurent artificiels et échangent des dialogues peu convaincants; les commentaires insérés dans la narration, séduisants au départ, se transforment en gloses explicatives inutiles. Et le dénouement du roman, qui devrait avoir une ampleur tragique, a les allures d'une farce grotesque. Le regard désirant et fiévreux de l'homme y rencontre l'Eil-sexe de l'Amazone, évidemment refait grâce aux bons soins du plasticien: "[un] sexe nouveau, serré et juvénile, sans poil, un sexe évoluant à travers toutes les étapes de la guérison, sexe croqué, sexe bouchée, une mordée» (188). Cette image, qui entrelace l'acharnement chirurgical, le rajeunissement obligé et une sexualité fétichiste, rend bien le travail d'hyperbolisation auquel s'adonne Nelly Arcan dans À ciel ouvert et témoigne, par là même, des dangers que peut comporter l'écriture d'un roman à la troisième personne. Comment passer de la conscience subjective à la mise en scène de personnages fictifs et autonomes en ne cédant pas à la duplication narcissique de soi? Cette question mérite, me semble-t-il, d'être posée.

Il s'avère presque impossible de tisser des liens entre Pourquoi faire une maison avec ses morts, À ciel ouvert et Ce n'est pas une façon de dire adieu ${ }^{4}$ de Stéfani Meunier,

$$
++
$$

4 Stéfani Meunier, Ce n'est pas une façon de dire adieu, Montréal, Boréal, 2007, 212 p. 
paru aux Éditions du Boréal. Ce dernier roman s'inscrit dans une tout autre perspective, adopte une poétique plus proche de celle d'Yvon Rivard, et s'éloigne du catastrophisme diffus mis en scène dans les deux textes précédents. Stéfani Meunier s'intéresse plus particulièrement aux relations entre trois êtres solitaires évoluant dans le New York de la fin des années 1960. Sa fin du monde renvoie à la fois à la fin d'une époque, celle des sixties, et à la fin de l'amitié unissant Ralf, Sean et Héloïse.

Ralf, jeune homme timide et introverti, héberge chez lui Sean, un musicien nomade en rupture avec sa famille bourgeoise de Westmount. Ils mènent une vie paisible jusqu'au jour où Héloïse entre dans la vie routinière de Ralf et menace l'équilibre précaire qu'il avait réussi à créer. Tout se passe comme si Ralf attirait malgré lui des êtres qui lui étaient à la fois complémentaires et opposés: avides de mouvement et de changement, Héloïse et Sean en viennent presque naturellement à s'éprendre l'un de l'autre. Loin des grands élans romantiques, Stéfani Meunier séparera les amoureux qui ne vivront leur idylle que par procuration.

L'intrigue se développe au gré des réflexions intimes des trois personnages qui se relaient, de chapitre en chapitre, assumant chacun leur tour la narration de leur histoire commune. Les courts chapitres portent souvent des titres s'inspirant de chansons populaires des années 1960 - celles des Beatles notamment, groupe adulé par Ralf - et ancrent le roman dans l'esprit d'une époque. La fin de l'amitié sera d'ailleurs associée à la dissolution des Beatles: «nous avions vraiment assimilé le fait que les Beatles étaient bel et bien séparés. La jeunesse, c'était fini. Le présent, c'était du passé. Quand les Beatles se sont séparés, ils ont inventé l'avenir. » (132)

Au-delà de cette présence emblématique de la musique - qui a parfois la valeur, mais pas nécessairement la profondeur, d'une forme de philosophie de l'existence - , le roman de Meunier s'attache à décrire la solitude de trois êtres, «trois îles» pour reprendre le titre d'un des derniers chapitres. Les îles sont d'abord métaphoriques: refuges, silences intérieurs, blessures, et symbolisent l'impossible communion des trois amis. Elles sont également incarnées en des lieux précis, New York bien sûr, mais surtout l'île des Caraỉbes où se retrouve Sean et où il aurait aimé se réfugier plus longtemps pour éviter d'affronter la réalité d'un amour interdit:

Et je me suis senti vide et perdu quand j'ai compris, dans l'avion, qu'elle ne serait plus devant moi, le lendemain, que je ne serais plus sur l'île, que c'était fini, qu'elle n'était plus là pour me protéger et me pousser au bas des falaises. Je me sentais comme un enfant qui lâche volontairement la main de sa mère pour se rendre compte tout de suite qu'elle a disparu. J'ai paniqué. Mais je n'ai rien dit. Mon héritage de l'île, ça, ne rien dire, parce que tout ce qu'il y aurait d'important à dire aurait l'air complètement ridicule une fois dit. C'est ça l'île. Et je me rappelle l'avoir pleurée aussi, moi qui n'avais pas encore, à l'époque, jamais vraiment pleuré. (162)

Ce bref passage contient en quelque sorte l'une des clés du roman : enfermés en euxmêmes, les trois personnages n'arrivent guère ou très rarement à saisir le moment présent, ils sont presque toujours décalés par rapport à leurs actes et à leurs idéaux, ils se regardent être. Paradoxalement peut-être, Meunier investit le moment et tente 
d'en livrer l'essence: les objets et les lieux, minutieusement racontés, s'offrent au lecteur qui, enveloppé par la lenteur de la narration, en ressent la présence.

Difficile de savoir ce qui nous éloigne de ces personnages et de ce style... Certes, le roman n'est pas sans failles (les dialogues, notamment, ont quelque chose de figé et certaines explications narratoriales semblent superflues), mais il ne présente pas non plus de défauts trop apparents. C'est peut-être sa lenteur qui en provoque l'épuisement ou son histoire, trop mince, qui ne réussit pas à convaincre. L'on sort malheureusement indemne de ce livre, inentamé, presque intouché, comme si les destins de Ralf, de Sean et d'Héloïse se contentaient d'effleurer celui ou celle qui en prenait connaissance. 